\title{
Impaired photosystem I oxidation induces STN7-dependent phosphorylation of the light-harvesting complex I protein Lhca4 in Arabidopsis thaliana
}

\author{
Anna Ihnatowicz $\cdot$ Paolo Pesaresi $\cdot$ Katharina Lohrig • \\ Dirk Wolters · Bernd Müller · Dario Leister
}

Received: 17 July 2007 / Accepted: 12 October 2007 / Published online: 30 October 2007

(C) Springer-Verlag 2007

\begin{abstract}
Reduction of the plastoquinone (PQ) pool is known to activate phosphorylation of thylakoid proteins. In the Arabidopsis thaliana mutants psadl-1 and psae1-3, oxidation of photosystem I (PSI) is impaired, and the PQ pool is correspondingly over-reduced. We show here that, under these conditions, the antenna protein Lhca4 of PSI becomes a target for phosphorylation. Phosphorylation of the mature Lhca4 protein at Thr16 is suppressed in $\operatorname{stn} 7$ psadl and $\sin 7$ psael double mutants. Thus, under extreme redox conditions, hyperactivation of thylakoid protein kinases and/or reorganization of thylakoid protein complex distribution increase the susceptibility of PSI to phosphorylation.
\end{abstract}

Anna Ihnatowicz and Paolo Pesaresi contributed equally to the article.

Electronic supplementary material The online version of this article (doi:10.1007/s00425-007-0650-8) contains supplementary material, which is available to authorized users.

\section{A. Ihnatowicz}

Abteilung für Pflanzenzüchtung und Genetik,

Max-Planck-Institut für Züchtungsforschung,

Carl-von-Linné-Weg 10, 50829 cologne, Germany

P. Pesaresi

Dipartimento di Produzione Vegetale,

Università Statale di Milano c/o Parco Tecnologico Padano

Via Einstein, Loc. Cascina Codazza, 26900 Lodi, Italy

K. Lohrig · D. Wolters

Abteilung für Analytische Chemie,

Ruhr-Universität Bochum, Universitätsstr. 150,

44780 Bochum, Germany

B. Müller · D. Leister $(\square)$

Lehrstuhl für Botanik, Department Biologie I,

Ludwig-Maximilians-Universität München,

Menzinger Str. 67, 80638 Munich, Germany

e-mail: leister@1rz.uni-muenchen.de
Keywords Lhca4 - Protein kinase $\cdot$ psael $\cdot$ psadl pThr . Phosphothreonine $\cdot$ STN7 $\cdot$ Thylakoid phosphorylation

\begin{tabular}{ll}
\multicolumn{2}{l}{ Abbreviations } \\
LHCI (II) & Light-harvesting complex I (II) \\
PQ & Plastoquinone \\
PSI (II) & Photosystem I (II) \\
WT & Wild type
\end{tabular}

\section{Introduction}

Photosynthetic organisms have evolved regulatory mechanisms that allow them to adapt their photosynthetic performance to changes in light conditions (Rochaix 2007). In the short term, this involves the phosphorylation of several core subunits of photosystem II (PSII), as well as some of its light-harvesting proteins (LHCII; Vener 2007). In flowering plants, the reversible phosphorylation of thylakoid proteins is regulated by light, temperature and redox state (Allen 1992; Bergantino et al. 1995; Vener et al. 1998; Aro and Ohad 2003). Changes in thylakoid protein phosphorylation have been associated with a number of regulatory and adaptive responses, including state transitions (Wollman 2001) and long-term photosynthetic acclimation (Bonardi et al. 2005). Technological improvements in the fields of chromatography and mass spectrometry have enhanced our ability to identify phosphoproteins within complex mixtures of polypeptides, and a number of novel thylakoid phosphoproteins have been described in the last few years (Vener 2007). The PSI-D1 subunit was the first phosphoprotein to be isolated from light-adapted PSI complexes (Hansson and Vener 2003). In the same work, the TMP14 protein was also shown to be phosphorylated. The latter was recently 
found to be a subunit of PSI and renamed PSI-P (Khrouchtchova et al. 2005).

Several homologous protein kinases involved in the phosphorylation of thylakoid proteins have been identified recently, namely, the thylakoid-associated Ser/Thr protein kinases STT7 in Chlamydomonas reinhardtii (Depege et al. 2003), and STN7 and STN8 in Arabidopsis thaliana (Bellafiore et al. 2005; Bonardi et al. 2005; Vainonen et al. 2005). STT7 and STN7 are required for the phosphorylation of LHCII proteins, whereas STN8 is necessary for modification of PSII subunits. However, the possibility that STN7 and STN8 do not directly phosphorylate antenna and PSII core proteins, respectively, but are components of partially overlapping phosphorylation cascades cannot be excluded (Bonardi et al. 2005).

The aim of our study was to identify novel thylakoid phosphoproteins, and to this purpose the photosynthetic mutants psadl-1 and psael-3 (Ihnatowicz et al. 2004, 2007) with a general and drastic increase in the phosphorylation of thylakoid proteins were analysed. In these mutants, the mature Lhca4 protein is phosphorylated near its N-terminus, at Thr16, and the analysis of $p \operatorname{sad} 1-1 \sin 7-1$ and psae1-3 stn7-1 double mutants implies that the phosphorylation depends on STN7.

\section{Materials and methods}

Plant materials

The A. thaliana mutants psad1-1, psae1-3 and stn7-1 have already been described (Ihnatowicz et al. 2004; Bonardi et al. 2005; Ihnatowicz et al. 2007). The lhca4-1 mutant, corresponding to the line Salk_138555 (ecotype Col-0), was identified by screening the insertion flanking database SIGnAL (http://signal.salk.edu/cgi-bin/tdnaexpress). Details of the T-DNA insertion and the primers used for segregation analysis are given in Supplementary Fig. 1. The double mutants psad1-1 stn7-1, psae1-3 stn7-1 and psae1-3 lhca41 were generated by crossing the corresponding single mutants and identifying homozygous F2 plants by PCR. Wild-type (WT) Arabidopsis thaliana (L.) Heynh., ecotype Columbia 0 (Col-0), and mutant plants were grown as described (Pesaresi et al. 2002) and, unless otherwise indicated, all analyses were performed on light-adapted plants at the eight-leaf rosette stage.

Protein complex isolation and immunoblot analysis

Leaves were harvested in the middle of the light period, and thylakoids were prepared as described previously (Bassi et al. 1985). For the isolation of PSI complexes, the membranes were washed twice with 5 mM EDTA ( $\mathrm{pH} 7.8$ ), centrifuged $\left(5 \mathrm{~min}, 10000 \mathrm{~g}\right.$ ) and resuspended in $\mathrm{ddH}_{2} \mathrm{O}$ to reach $2 \mathrm{mg} / \mathrm{ml}$ of total chlorophyll concentration. After solubilization with $2 \%(\mathrm{w} / \mathrm{v}) \beta$-dodecyl maltoside $\left(10 \mathrm{~min}, 4^{\circ} \mathrm{C}\right)$, PSI isolation and fractionation by $16-23 \%$ gradient SDSPAGE was carried out as described before (Jensen et al. 2000) and proteins were visualized by Coomassie Blue staining. Western analysis of thylakoid proteins (for phosphothreonine) or PSI complexes (for phospothreonine and Lhcb2) was performed as reported (Pesaresi et al. 2002) and signals were detected using the Enhanced Chemiluminescence Western Blotting Kit (Amersham Biosciences).

Phosphopeptide analysis

PSI complexes were subjected to proteolysis with trypsin and chymotrypsin (overnight at $37^{\circ} \mathrm{C}$ ) and undigested proteins were removed by ultrafiltration (Microcon YM-3, Millipore). The phosphorylated and non-phosphorylated peptides at the Thr residue were obtained from PSL GmbH (Heidelberg, Germany).

Purified samples were analysed by Multidimensional Protein Identification Technology (MudPIT) (for details see Supplementary Methods). MS/MS data were analysed with the SEQUEST algorithm (Eng et al. 1994). To ensure reliable protein identification, only peptides with a $\Delta \mathrm{Cn}$ score $>0.1$ were considered. In addition, a peptide had to be partially tryptic or chymotryptic to be accepted and the cross-correlation scores of single, double and triple charged peptides had to be $>1.8,>2.5$ and $>3.5$, respectively. Peptides were manually evaluated as described elsewhere (Washburn et al. 2001).

\section{Results}

A novel 22-kDA phosphoprotein is present

in psad1-1 and psae 1-3 thylakoids

Because phosphorylation of thylakoid proteins is increased under conditions, which augment the reduction of the plastoquinol pool (Aro and Ohad 2003), the PSI mutants psad1-1 and psae1-3, both of them showing a reduction by about $60 \%$ of the subunits of the stromal ridge of PSI (Varotto et al. 2000; Ihnatowicz et al. 2004, 2007), were examined for the presence of novel thylakoid phosphoproteins. As expected, phosphorylation of PSII and LHCII proteins was markedly increased in light-adapted psadl-1 and psael-3 thylakoids, as revealed by Western analysis with an antibody specific for phosphothreonine (pThr) residues (Fig. 1). In addition to thylakoid phosphoproteins with apparent molecular weights between 8 and $20 \mathrm{kDa}$, which include PSI-P (Hansson and Vener 2003; Khrouchtchova et al. 2005) and TSP9 (Carlberg et al. 2003), a novel species of about $22 \mathrm{kDa}$ appears in the mutant thylakoids (see asterisk in Fig. 1). 


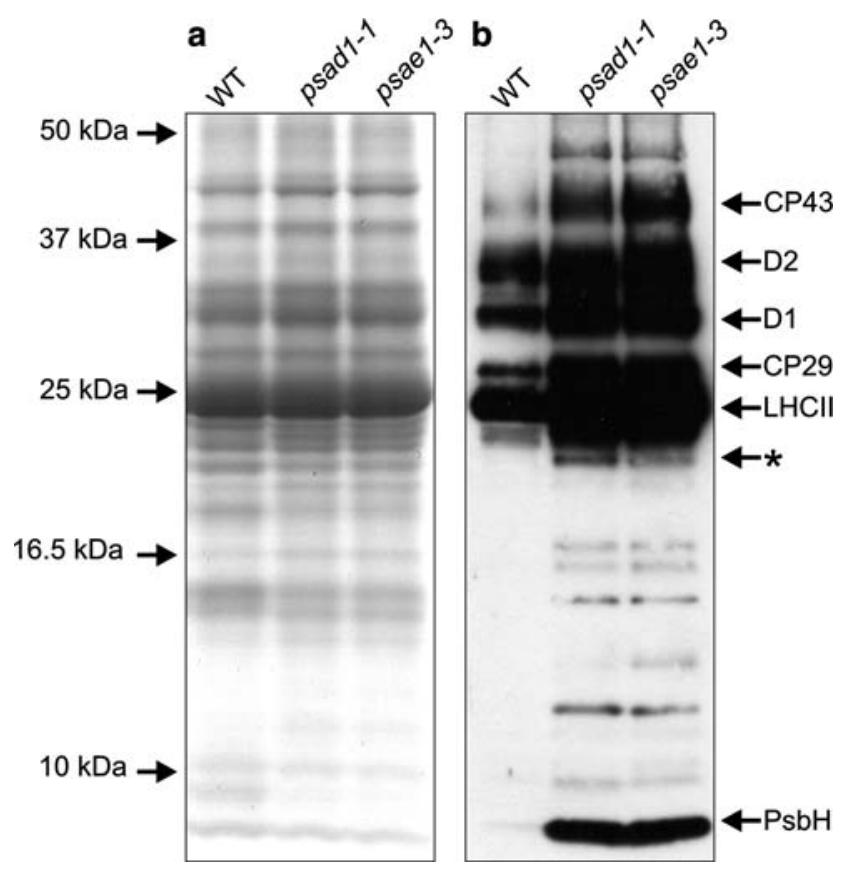

Fig. 1 Thylakoid protein phosphorylation. a Identical amounts of thylakoid proteins corresponding to $5 \mu \mathrm{g}$ of total chlorophyll from WT, psad1-1 and psae 1-3 leaves were fractionated by SDS-PAGE and visualized by Coomassie Blue staining. Note that only the portion of the Coomassie-stained gel containing phosphoproteins is shown. b Immunolabelling was performed with a pThr-specific antibody. The results shown are representative of those obtained in three independent experiments

Phosphorylation of the novel PSI phosphoprotein depends on STN7

Because the migration behaviour of the 22-kDa phosphoprotein resembles that of PSI antenna proteins (LHCI), PSI complexes were isolated from WT, psad1-1 and psael-3 leaves, fractionated by SDS-PAGE (Fig. 2a), and subjected to Western analyses with pThr- (Fig. 2b) and Lhcb2-specific antibodies (Fig. 2c). In addition to the highly phosphorylated LHCII fraction (pLHCII) described before (Pesaresi et al. 2002), the 22-kDa phosphoprotein, which comigrates with Lhcal and Lhca4, was found to be associated with PSI in psad1-1 and psae1-3, but not in WT leaves (Fig. 2).

To test whether phosphorylation of the novel PSI protein depends on the kinase STN7, the double mutants psad1-1 $\operatorname{stn} 7-1$ and psae1-3 $\operatorname{stn} 7-1$ were generated and their PSI phosphorylation patterns were analysed as above. The novel PSI phosphoprotein was not detectable in the double mutants (Fig. 2), implying that its phosphorylation requires STN7 activity. The pLHCII-PSI complex is also absent in the double mutants, which supports the notion that phosphorylation of LHCII is essential for its interaction with PSI (Pesaresi et al. 2002).

The N-terminal portion of Lhca4 is phosphorylated at Thr16

To identify the novel PSI phosphoprotein, PSI complexes isolated from psadl-1 and psae1-3 thylakoid membranes were subjected to proteolysis with trypsin and chymotrypsin, and analysed by automated multidimensional protein identification technology (MudPIT), which combines biphasic liquid chromatography with electrospray ionization tandem mass spectrometry (MS/MS). In both psae1-3 (Fig. 3a) and psadl-1 (Supplementary Fig. 2), but not in WT samples, the phosphorylated peptide sequence TGSLAGDNGFDPLGLAEDPENLK, which originates from Lhca4, was identified with a cross correlation value of 4.15 for the doubly charged fragment ion, where the $b$ - and $y$-ion series showed close-to-complete coverage of this peptide. The $\mathrm{m} / \mathrm{z}$ ratio of the precursor ion selected for MS/
Fig. 2 PSI phosphorylation. a Identical amounts of PSI complexes isolated from WT and mutant (psae1-3 stn7-1, psad1-1 stn7-1, psae1-3, psad1-1) leaves were fractionated by SDSPAGE and visualized by Coomassie Blue staining. Positions of subunits previously identified by immunodetection (Jensen et al. 2000) are indicated. b Western analyses for pThr residues were performed on identical amounts of PSI complexes derived from WT and mutant leaves. c A similar filter to that shown in (b), but probed with an Lhcb2-specific antibody

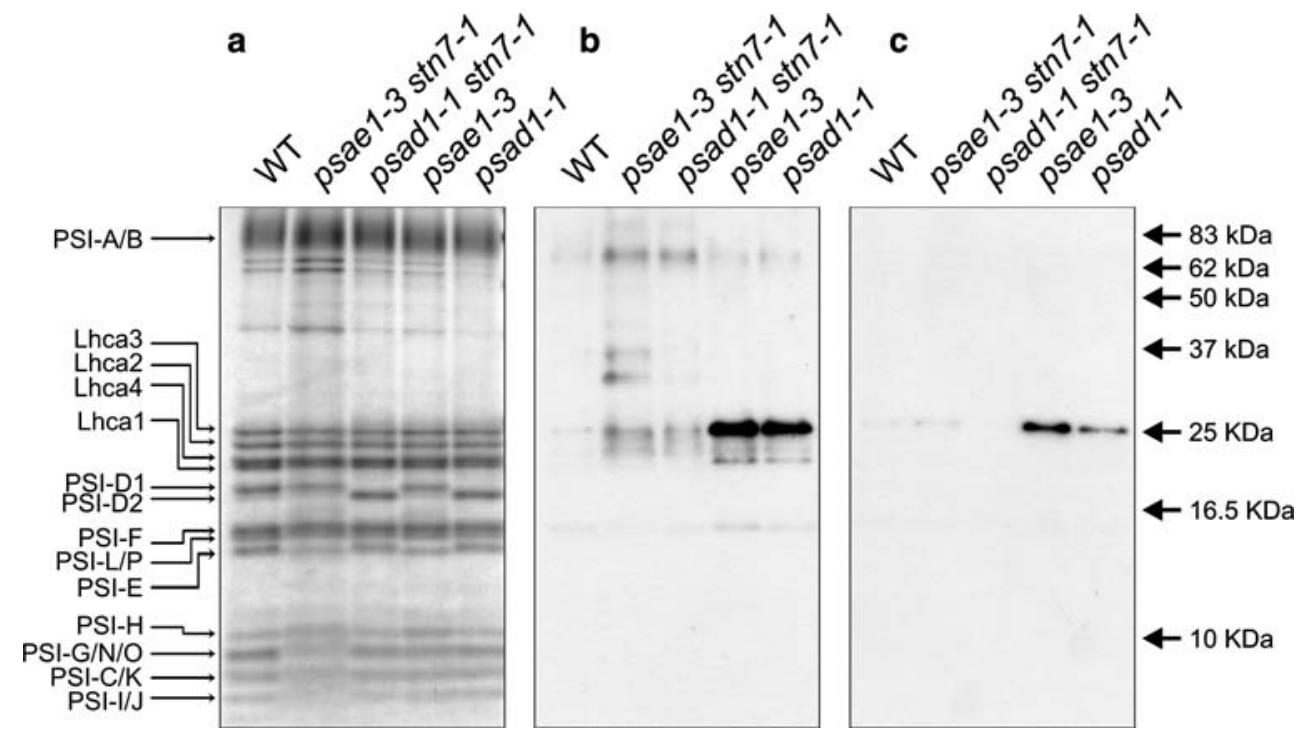


$2322212019181716151413121110987654321 \%$

t G SLAGDNGFDPLGLAEDPENLK

b 1 234567891011121314151617181920212223
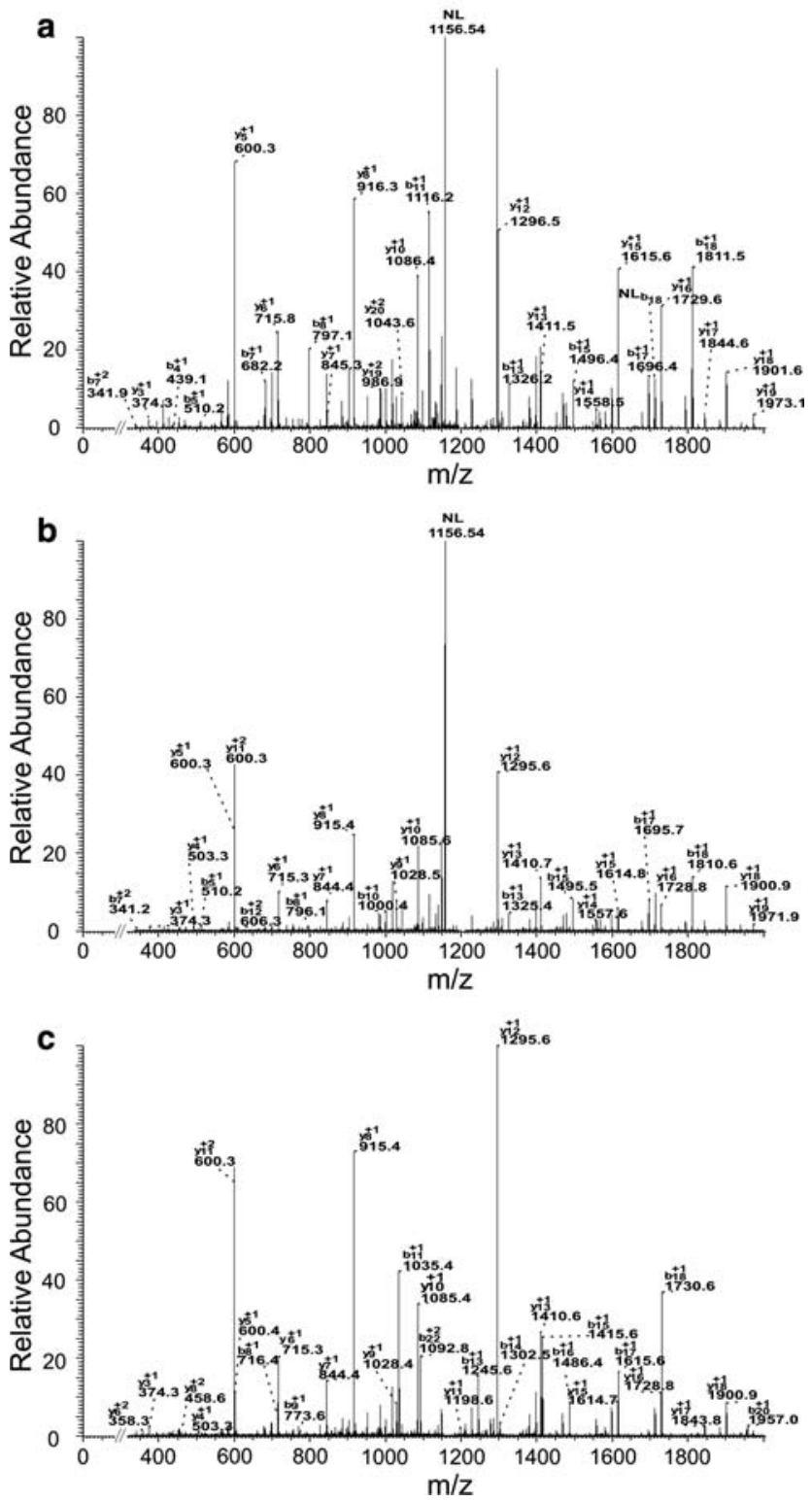

Fig. 3 Sequencing of a phosphopeptide released, by proteolysis with trypsin and chymotrypsin, from PSI complexes isolated from psae1-3 leaves. a Fragmentation spectrum (MS2) of the doubly protonated and phosphorylated peptide from psae1-3. b MS2 spectrum of the synthesized phosphorylated peptide tGSLAGDNGFDPLGLAEDPENLK. c MS2 spectrum of the synthesized non-phosphorylated peptide TGSLAGDNGFDPLGLAEDPENLK. Note that no neutral loss of $\mathrm{H}_{3} \mathrm{PO}_{4}(\mathrm{NL} ; m / z=-49)$ is observed. The $\mathrm{b}$ - and $\mathrm{y}$-ions are indicated in the spectra and in the corresponding peptide sequence. The peptide fragment that underwent neutral loss of $\mathrm{H}_{3} \mathrm{PO}_{4}$ is indicated as $N L$. The lowercase $\mathbf{t}$ designates $\mathrm{pThr}$

MS analysis was $1,206.8$, which was 0.63 Da higher than expected. However, for database search algorithms like SEQUEST, an error of \pm 1 to $2 \mathrm{Da}$ is tolerable for reliable characterization of peptides.
In addition, the typical neutral loss (NL) of a phosphorylated peptide was obvious in the spectrum (see peak indicated as "NL"). These data, together with the fact that the novel phosphoprotein could be detected with a pThr-specific antibody (see Figs. 1 and 2) but not with an antibody specific for phosphoserine (data not shown), imply that Lhca4 is indeed phosphorylated at Thr16 in psadl-1 and psae 1-3 plants. To further investigate this aspect, the Lhca4 peptide was synthesized in its threonine-phosphorylated (Fig. 3b) and threonine-non-phosphorylated forms (Fig. 3c). The MS/MS spectrum of the phosphorylated peptide is very similar to the spectrum shown in Fig. 3a and the predominant NL of the phosphate group (-49) is characteristic for the phosphorylated peptide. In contrast, the non-phosphorylated peptide shows no NL. Since the b-ion series is not as predominant as the y-ion series, only very little but still some NL loss is observed from fragment ions (e.g. predominant $\mathrm{b}_{18} \mathrm{~m} / \mathrm{z}=1812$ shows -98 loss at $\mathrm{m} / \mathrm{z}=1714$; Fig. 3a and Supplementary Fig. 2).

To confirm that the novel phosphoprotein detected corresponds to pLhca4, an insertion mutant for LHCA4 was isolated and crossed to psae1-3 plants to generate the double mutant psae1-3 lhca4-1. Western analysis of thylakoid protein preparations from WT, psae1-3 and psae1-3 lhca4-1 plants with a pThr-specific antibody showed that thylakoid membranes isolated from the double mutant resembled those of psae1-3, except for the specific absence of the novel phosphoprotein (Fig. 4). This strongly suggests that pLhca4 is the novel phosphoprotein that accumulates in an STN7-dependent manner in the mutants psadl-1 and psae1-3. The possibility that Lhcal is also phosphorylated

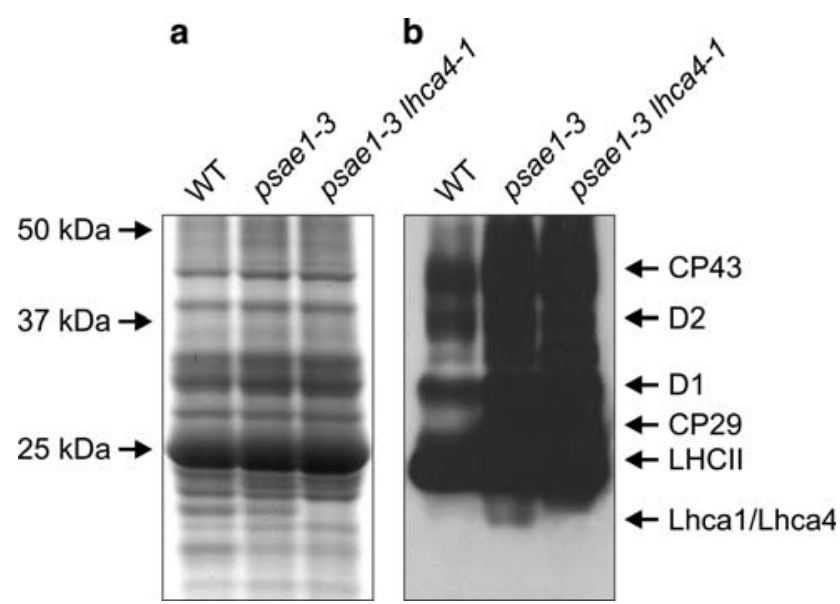

Fig. 4 Thylakoid protein phosphorylation in WT, psae1-3 and psae13 lhca4-1 leaves. a Identical amounts of thylakoid proteins (corresponding to $5 \mu \mathrm{g}$ of total chlorophyll) from WT, psae1-3 and psae 1-3 lhca $4-1$ leaves were fractionated by SDS-PAGE and visualized by Coomassie Blue staining. Note that only the portion of the Coomassiestained gel containing phosphoproteins is shown. b Western analysis was performed with a pThr-specific antibody. The results shown are representative of those obtained in three independent experiments 
Depege N, Bellafiore S, Rochaix JD (2003) Role of chloroplast protein kinase Stt7 in LHCII phosphorylation and state transition in Chlamydomonas. Science 299:1572-1575

Eng JK, McCormack AL, Yates JRI (1994) An approach to correlate tandem mass spectral data of peptides with amino acid sequences in a protein database. J Am Soc Mass Spectrum 5:976-989

Hansson M, Vener AV (2003) Identification of three previously unknown in vivo protein phosphorylation sites in thylakoid membranes of Arabidopsis thaliana. Mol Cell Proteomics 2:550-559

Ihnatowicz A, Pesaresi P, Leister D (2007) The E subunit of photosystem I is not essential for linear electron flow and photoautotrophic growth in Arabidopsis thaliana. Planta 226:889-895

Ihnatowicz A, Pesaresi P, Varotto C, Richly E, Schneider A, Jahns P, Salamini F, Leister D (2004) Mutants for photosystem I subunit D of Arabidopsis thaliana: effects on photosynthesis, photosystem I stability and expression of nuclear genes for chloroplast functions. Plant J 37:839-852

Jensen PE, Gilpin M, Knoetzel J, Scheller HV (2000) The PSI-K subunit of photosystem I is involved in the interaction between lightharvesting complex I and the photosystem I reaction center core. J Biol Chem 275:24701-24708

Khrouchtchova A, Hansson M, Paakkarinen V, Vainonen JP, Zhang S, Jensen PE, Scheller HV, Vener AV, Aro EM, Haldrup A (2005) A previously found thylakoid membrane protein of $14 \mathrm{kDa}$ (TMP14) is a novel subunit of plant photosystem I and is designated PSI-P. FEBS Lett 579:4808-4812

Klimmek F, Ganeteg U, Ihalainen JA, van Roon H, Jensen PE, Scheller HV, Dekker JP, Jansson S (2005) Structure of the higher plant light harvesting complex I: in vivo characterization and structural interdependence of the Lhca proteins. Biochemistry 44:30653073

Pesaresi P, Lunde C, Jahns P, Tarantino D, Meurer J, Varotto C, Hirtz RD, Soave C, Scheller HV, Salamini F, Leister D (2002) A stable LHCII-PSI aggregate and suppression of photosynthetic state transitions in the psae1-1 mutant of Arabidopsis thaliana. Planta 215:940-948

Rochaix JD (2007) Role of thylakoid protein kinases in photosynthetic acclimation. FEBS Lett 581:2768-2775

Vainonen JP, Hansson M, Vener AV (2005) STN8 protein kinase in Arabidopsis thaliana is specific in phosphorylation of photosystem II core proteins. J Biol Chem 280:33679-33686

Varotto C, Pesaresi P, Meurer J, Oelmuller R, Steiner-Lange S, Salamini F, Leister D (2000) Disruption of the Arabidopsis photosystem I gene $p s a E 1$ affects photosynthesis and impairs growth. Plant J 22:115-124

Vener AV (2007) Environmentally modulated phosphorylation and dynamics of proteins in photosynthetic membranes. Biochim Biophys Acta 1767:449-457

Vener AV, Ohad I, Andersson B (1998) Protein phosphorylation and redox sensing in chloroplast thylakoids. Curr Opin Plant Biol 1:217-223

Washburn MP, Wolters D, Yates JR 3rd (2001) Large-scale analysis of the yeast proteome by multidimensional protein identification technology. Nat Biotechnol 19:242-247

Wollman FA (2001) State transitions reveal the dynamics and flexibility of the photosynthetic apparatus. EMBO J 20:3623-3630 